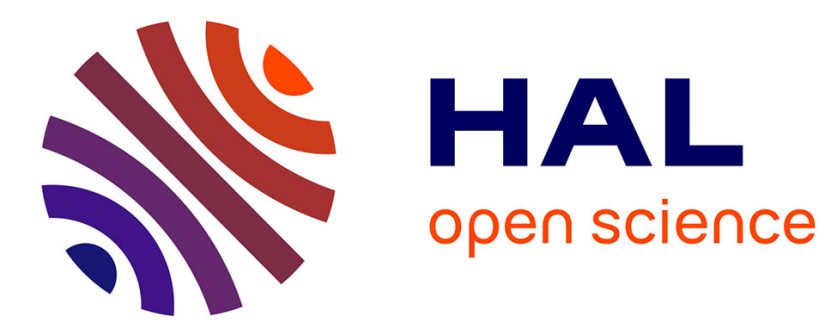

\title{
Structure of self-gravity wakes in Saturn's A ring as measured by Cassini CIRS
}

\author{
C. Ferrari, S. Brooks, S. Edgington, C. Leyrat, S. Pilorz, L. Spilker
}

\section{To cite this version:}

C. Ferrari, S. Brooks, S. Edgington, C. Leyrat, S. Pilorz, et al.. Structure of self-gravity wakes in Saturn's A ring as measured by Cassini CIRS. Icarus, 2008, 199 (1), pp.145. 10.1016/j.icarus.2008.09.001 . hal-00499094

\section{HAL Id: hal-00499094 https://hal.science/hal-00499094}

Submitted on 9 Jul 2010

HAL is a multi-disciplinary open access archive for the deposit and dissemination of scientific research documents, whether they are published or not. The documents may come from teaching and research institutions in France or abroad, or from public or private research centers.
L'archive ouverte pluridisciplinaire HAL, est destinée au dépôt et à la diffusion de documents scientifiques de niveau recherche, publiés ou non, émanant des établissements d'enseignement et de recherche français ou étrangers, des laboratoires publics ou privés. 


\section{Accepted Manuscript}

Structure of self-gravity wakes in Saturn's A ring as measured by Cassini CIRS

C. Ferrari, S. Brooks, S. Edgington, C. Leyrat, S. Pilorz, L. Spilker

PII: S0019-1035(08)00318-7

DOI: $\quad$ 10.1016/j.icarus.2008.09.001

Reference: $\quad$ YICAR 8758

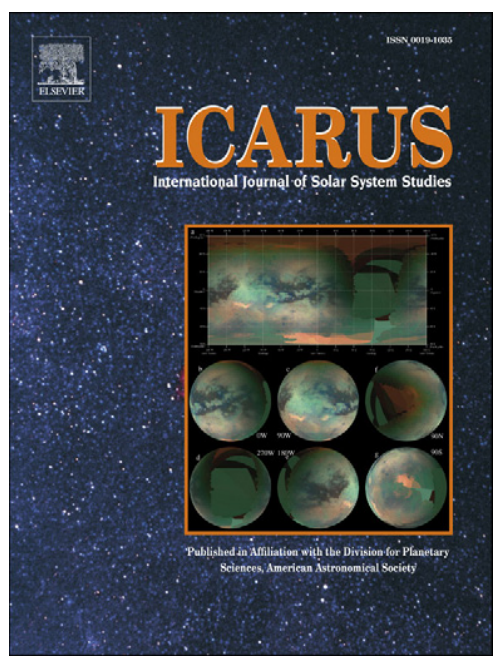

To appear in: Icarus

Received date: 4 February 2008

Revised date: 8 September 2008

Accepted date: 12 September 2008

Please cite this article as: C. Ferrari, S. Brooks, S. Edgington, C. Leyrat, S. Pilorz, L. Spilker, Structure of self-gravity wakes in Saturn's A ring as measured by Cassini CIRS, Icarus (2008), doi:

10.1016/j.icarus.2008.09.001

This is a PDF file of an unedited manuscript that has been accepted for publication. As a service to our customers we are providing this early version of the manuscript. The manuscript will undergo copyediting, typesetting, and review of the resulting proof before it is published in its final form. Please note that during the production process errors may be discovered which could affect the content, and all legal disclaimers that apply to the journal pertain. 


\title{
Structure of self-gravity wakes in Saturn's A ring as measured by Cassini CIRS
}

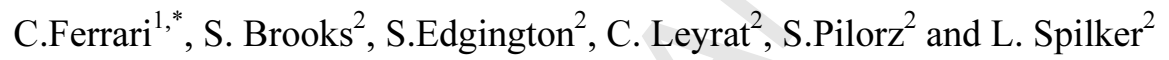 \\ ${ }^{1}$ CEA, IRFU, AIM, F-91191 Gif-sur-Yvette, France \\ ${ }^{1}$ Université Paris Diderot Paris7, Laboratoire AIM, F-75205 Paris cedex 13, France \\ * Corresponding author E-Mail address : cferrari@cea.fr \\ 2 Jet Propulsion Laboratory/ NASA, Pasadena, California 91109
}

Pages : 25

Tables : 2

Figures : 4 
Proposed running head: A ring self-gravity wakes observed by CIRS

\section{Editorial correspondence to:}

Pr. C. Ferrari

CEA, IRFU, SAp

Bat. 709, Orme des Merisiers

91191 Gif sur Yvette Cedex, France

Tel: +33169087829

Fax : +33169086577

E-mail address: cferrari@cea.fr 


\section{ABSTRACT}

The CIRS infrared spectrometer onboard the Cassini spacecraft has scanned Saturn's A ring azimuthally from several viewing angles since its orbit insertion in 2004. A quadrupolar asymmetry has been detected in this ring at spacecraft elevations ranging between $16^{\circ}$ to $37^{\circ}$. Its fractional amplitude decreases from $22 \%$ to $8 \%$ from $20^{\circ}$ to $37^{\circ}$ elevations. The patterns observed in two almost complete azimuthal scans at elevations $20^{\circ}$ and $36^{\circ}$ strongly favor the self-gravity wakes as the origin of the asymmetry. The elliptical, infinite cylinder model of Hedman et al. (2007) can reproduce the CIRS observations well. Such wakes are found to have an average height-to-spacing ratio $\mathrm{H} / \lambda=0.1607 \pm 0.0002$, a width-over-spacing $\mathrm{W} / \lambda=0.3833 \pm$ 0.0008. Gaps between wakes, which are filled with particles, have an optical depth $\tau_{\mathrm{G}}=0.1231 \pm$ 0.0005 . The wakes mean pitch angle $\Phi_{\mathrm{W}}$ is $70.70^{\circ} \pm 0.07^{\circ}$, relative to the radial direction. The comparison of ground-based visible data with CIRS observations constrains the A ring to be a monolayer. For a surface mass density of $40 \mathrm{~g} . \mathrm{cm}^{-2}$ (Tiscarino et al. 2007), the expected spacing of wakes is $\lambda \approx 60 \mathrm{~m}$. Their height and width would then be $\mathrm{H} \approx 10 \mathrm{~m}$ and $\mathrm{W} \approx 24 \mathrm{~m}$, values that match the maximum size of particles in this ring as determined from ground-based stellar occultations (French and Nicholson 2000).

Keywords: Planetary rings - Infrared observations - Saturn, Rings - Disks 


\section{Introduction}

The so-called self-gravity wakes are ephemeral aggregates of ring particles that form in the outer parts of Saturn's rings, where clumping due to mutual gravity between particles can resist destructive collisions and keplerian shear. Numerical simulations of the local dynamics in the A ring, including collisions and self-gravity, show that for most probable ring surface densities and particle volume densities, the wakes are elongated, having a pitch angle of about $69^{\circ}$ with the local radial direction (Salo 1992, 1995, Daisaka and Ida 1999). Their typical spacing, or wavelength, is proportional to the ring surface density and is expected to be about 50 -to- 100 meters in this ring. The typical height-over-wavelength ratio $\mathrm{H} / \lambda$ observed in these simulations is in the range $0.17-0.25$ and the width-over-wavelength ratio $\mathrm{W} / \lambda$ between 0.25 and 0.33 typically (French et al., 2007).

An azimuthal asymmetry of the solar reflected light by Saturn's A ring was first observed from ground (Camichel 1958). Colombo et al. (1976) were the first to propose the self-gravity wakes as the origin of this asymmetry. The ring brightness peaks where the wakes are perpendicular to the line of sight and the gaps in-between them are invisible. It becomes minimal as the wakes are parallel to the line of sight and the relative empty gaps are no longer hidden. This forms an azimuthal brightness asymmetry with two minima and two maxima, know as the quadrupolar asymmetry of the A ring. The asymmetry amplitude has been found to increase when Earth elevation above the ring plane increases from 0 to $12^{\circ}$, reaching here a maximum relative amplitude of $\sim 30 \%$. At higher elevations, the amplitude decreases to reach $10 \%$ at an elevation of $26^{\circ}$ (Thompson 1982, Lumme and Irvine 1976, 1979, Lumme et al. 1977, Thompson et al. 1981, 
French et al. 2007). The average pitch angle was found to be in the range $70^{\circ}-75^{\circ}$. The Voyager spacecraft also observed the quadrupolar asymmetry at an elevation of about $12.5^{\circ}$ (Franklin et al. 1987, Dones et al. 1993). The relative amplitude was found to reach a maximum of $\sim 33 \%$. The azimuthal variation was clearly identified as non-sinusoidal and the average pitch angle was found to be $\sim 70^{\circ}$. The quadrupolar asymmetry has also been detected more recently from ground at microwave wavelengths (Dunn et al. 2004) and in the radar echo of Saturn's rings (Nicholson et al. 2005). The numerical simulations have provided realistic spatial distributions of ring particles, particularly of self-gravity wakes. First ray-tracing experiments in these distributions confirmed the self-gravity wakes at the origin of the azimuthal asymmetry observed in visible light (Porco et al. 2001, Salo et al. 2004).

During the first four years of the mission, all remote sensing instruments onboard the Cassini spacecraft have detected the A ring quadrupolar asymmetry, from ultraviolet to radio wavelengths, from very different viewing angles, by very diverse ways. Observations of stellar and radio occultations by the rings led to important results on the radial variations of the wakes properties (Colwell et al. 2006, Hedman et al. 2007, Marouf et al. 2005). Azimuthal scans with the ISS cameras (Weiss et al. 2006) or the CIRS infrared spectrometer (Ferrari et al. 2005) fully sampled longitudinally the quadrupolar asymmetry at an average distance of $129,000 \mathrm{~km}$ from the planet.

The CIRS instrument provides a new window in the infrared domain to observe the quadrupolar asymmetry via the ring thermal emission. This emission is directly proportional to the apparent ring filling factor, which depends on the direction of the wakes relative to the line of sight. All azimuthal scans show the quadrupolar asymmetry with an amplitude that varies with the 
spacecraft elevation $\mathrm{B}$, between $16^{\circ}$ and $37^{\circ}$. In section 2 , the azimuthal variations of the $\mathrm{A}$ ring filling factor are presented as a function of the viewing geometry. A detailed study of the A ring temperature dependence with viewing geometry and its implications on the ring physical properties are reported in another paper (Leyrat et al, in preparation). In section 3, the morphology of self-gravity wakes is determined by fitting to CIRS data the morphological parameters of analytical wake models, as proposed by Hedman et al. (2007) and Colwell et al. (2006). These new constraints on the wakes' structure are finally compared with results obtained recently by the other Cassini instruments or by ground-based telescopes.

\section{Observations}

The CIRS instrument detects almost all the thermal radiation of Saturn's rings emitted in the infrared domain, between 7 to $1000 \mu \mathrm{m}$ (Flasar et al. 2004). The peak emission can be observed in its focal plane detector FP1, between 17 and $1000 \mu \mathrm{m}$. In this window, ring particles thermal properties, composition and local dynamics can be explored (Spilker et al. 2003). The ring thermal emission $I_{v}$ can be first approximated with a Planck function $B_{v}\left(T_{P}\right)$, weighted with a factor $\beta_{v}$, i.e. $I_{v}=\beta_{v} B_{v}\left(T_{P}\right)$, where $T_{P}$ is the ring effective temperature within the instrument field of view. The $\beta_{v}$ factor is a complex function of the ring filling factor, which is controlled by the ring vertical structure and density, and of the particles emissivity $\varepsilon_{v}$. As a first guess, the $\beta_{v}$ factor is assumed to be constant against wavenumber, i.e. $\beta_{v}=\beta$. This is true in the wavelength domain where most of the thermal emission happens (Spilker et al. 2005). If the ring is not isothermal within the field of view, this factor also includes residuals due to superimposed blackbody emissions (Ferrari and Leyrat 2006, Altobelli et al. 2007). It will be shown that in the present data 
set, this factor appears to be independent on phase angle. Also the dependence of $\beta$ with elevation B will be determined empirically.

\subsection{Data overview and processing}

Azimuthal scans of the A ring were obtained between July 2004 and the latest ' $180^{\circ}$-transfer' period ending on June 2007. During this long time period, the ring was scanned from different viewing points at a constant ring radius of $129,000 \mathrm{~km}$, in the middle of the ring (Spilker et al. 2003, Flasar et al. 2004, Ferrari et al. 2005, Leyrat et al. 2008). Thirteen scans are analyzed here (Table 1). In this time period, the orbital design favored the observation of the rings on their south lit face at low and mid phase angles and on their north unlit face at high phase angles. The A ring was scanned at average spacecraft elevations $B_{m}$ ranging from $-37^{\circ}$ to $+37^{\circ}$ and average phase angles $\alpha_{\mathrm{m}}$ between $15^{\circ}$ to $160^{\circ}$. All along this data collection, the Sun elevation below the ring plane regularly decreased from $\mathrm{B}^{\prime}=-24.5^{\circ}$ to $\mathrm{B}^{\prime}=-12.5^{\circ}$.

$* * * * * * * * * * * * * * *$ Insert Table 1

Spectra were obtained at a low spectral resolution of $15 \mathrm{~cm}^{-1}$ and calibrated at Goddard Space Flight Center by the CIRS calibration team using the procedure described in Flasar et al. (2004). They were averaged by series of $\mathrm{N}=10$ spectra along azimuth to reduce noise in the data. The dispersion on data points at each wavenumber $v$ of the average spectrum is the standard deviation on this average. This observed deviation is twice to ten times as large as the expected instrument noise spectral radiance (NESR) divided by $\sqrt{ } \mathrm{N}$ (this instrumental noise is inversely proportional 
to the square-root of the integration time, Flasar et al. 2004). The typical noise on the average spectra is about $4.10^{-9} \mathrm{~W} \cdot \mathrm{cm}^{-2} \cdot \mathrm{cm} \cdot \mathrm{sr}^{-1}$. Least-squares fits were performed on each average spectrum using a Levenberg-Marquardt minimization algorithm to recover $\beta_{v}$ and $\mathrm{T}_{\mathrm{P}}$. The MPFIT software package provided online by C. Markwardt at GSFC was used. The fit is limited to the wavenumber range of $40-400 \mathrm{~cm}^{-1}$, where the $\beta_{v}=\beta$ assumption is respected. Standard deviations on fitted temperature $T_{P}$ and factor $\beta$ have median values below $0.35 \mathrm{~K}$ and 0.01 respectively.

Azimuthal scans in the thermal infrared usually display features as a function of the local time, as the radiation is mainly dependent on the solar heating source. The phenomenon reported here is mainly dependent on the position of the observer relatively to the ring intercept point. Azimuthal scans are thus displayed in a longitudinal system where $\Phi$ denotes the spacecraft longitude relative to the ring radial direction at the ring intercept (equivalent to longitude $\Phi_{0}$ in Salo et al. (2004), fig. 6). This longitude at the ring intercept point can be approximated from usual ephemerides data, like the longitude $\theta$ relatively to the spacecraft longitude, the spacecraft elevation B, the distance of the spacecraft to Saturn's center $r_{S C}$, and the planet distance a, by the expression: $\Phi \sim-\left(\theta+\mathrm{a}^{*} \sin \theta / \mathrm{r}_{\mathrm{SC}} \cos \mathrm{B}\right)$.

$* * * * * * * * * * * * * * *$ Insert Figure 1

2.2 Azimuthal variations of the $\beta$ factor 
The azimuthal variations in the A ring of the ring emissivity $\beta(\Phi, \mathrm{B})$ are found to exhibit a very peculiar behavior (Fig. 1). They are large and do appear in all the scans, on lit $\left(\mathrm{B}_{\mathrm{m}}<0\right)$ and unlit faces $\left(B_{m}>0\right)$. Single scans do not cover the whole circumference but altogether they clearly show the quadrupolar asymmetry. These data are the first detection ever in the infrared domain of the A ring asymmetry (Ferrari et al. 2005). The $\beta$ factors vary between 0.5 and 0.75 depending on the spacecraft elevation. The peak-to-peak amplitude of the asymmetry decreases with increasing spacecraft elevation, i.e. about 0.16 at $\left|\mathrm{B}_{\mathrm{m}}\right|=22^{\circ}$ to only about 0.07 at $\left|\mathrm{B}_{\mathrm{m}}\right|=37^{\circ}$. At similar elevations, phase angle appears to be of little influence on $\beta(\Phi)$. This factor is expected to include effects of ring filling factor and inter-particle screening, which are linked with the spatial distribution of particles and are primarily function of the observer's elevation. If the particles are not isothermal, $\beta(\Phi)$ may also depend on the phase angle, all the most at angles where the thermal emission in the field of view is the superposition of two blackbodies at very different temperatures, i.e $I_{v}=\beta_{1} B_{v}\left(T_{1}\right)+\beta_{2} B_{v}\left(T_{2}\right)$ instead of $I_{v}=\beta B_{v}\left(T_{P}\right) . \beta(\Phi)$ would then include an error made by approximating the intensity to one blackbody emission curve. The little influence of phase angle on the $\beta(\Phi)$ observed here may indicate that the thermal contrast at the surface of particles/wakes is low. This point should be studied in more detail together with the thermal behaviour of this ring.

In a few scans, variations exhibit a very specific behavior, as illustrated by the flat appearance of the profile at $\left(\alpha_{m}, B_{m}\right)=\left(160^{\circ}, 26^{\circ}\right)$ or the very steep slope for $\left(\alpha_{m}, B_{m}\right)=\left(15^{\circ},-26^{\circ}\right)$, both around $\Phi=60^{\circ}$. The amplitudes of maxima located around $\Phi=30^{\circ}$ vary significantly with spacecraft mean elevation. These maxima appear to be of less amplitude than the ones located around $\Phi=200^{\circ}$, for similar mean elevation. These behaviors are mainly due to the variation of the spacecraft 
elevation around its mean value $B_{m}$ during the scans. An azimuthal variation of $\beta$ may be caused by a variation of the ring optical depth or by a change in the spacecraft elevation during the scan. Longitudinal variations of optical depth can be actual (as expected if wakes are present) or apparent (if they result from variations of the mean optical depth of the ring within the field of view as its footprint on the ring plane changes during the scan). The azimuthal profiles have been performed in the middle of the A ring, at a distance $\mathrm{a}=129,000 \mathrm{~km}$ from Saturn center, where the ring exhibits very little radial variation in optical depth at such a large scale. The spacecraft was usually indeed far away and the resulting spatial resolution of the FP1 3.9-mrad-wide field of view was rather low, varying from $2000 \mathrm{~km}$ to $6000 \mathrm{~km}$ per footprint. Optical depth variations were estimated for each footprint using radial profiles of optical depth as measured by the Voyager PPS instrument (Lane et al. 1982). They were found negligible compared to the observed variations of the $\beta$ factor. The spacecraft elevation and the spacecraft distance are known from ephemerides at any time during the scan. Amplitude variations are clearly correlated with changes in the spacecraft elevation B and longitude $\Phi$.

At this stage, every observed $\beta(\Phi, \mathrm{B})$ factor, as displayed in figure 1 , may be directly compared with a modeled $\beta$ factor of a ring structured with self-gravity wakes, in order to constrain their shape and spacing. This will be done in section 3 where some explicit expressions of $\beta(\Phi, B)$ are developed. Before that, in order to visualize the actual influence of the longitude $\Phi$ alone on $\beta$ and to obtain profiles as complete as possible along azimuth, azimuthal profiles at constant observer elevations $\mathrm{B}_{\mathrm{F}}$ are built from available data. Adding profiles together after they have been linearly interpolated from changing elevation $\mathrm{B}$ during the scan to fixed elevation $\mathrm{B}_{\mathrm{F}}$ does this. 
$* * * * * * * * * * * * * * *$ Insert Figure 2

2.3 Correction for varying viewing geometry during and among azimuthal scans

The purpose here is to obtain an azimuthal profile of $\beta\left(\Phi, \mathrm{B}_{\mathrm{F}}\right)$ at a constant spacecraft elevation $\mathrm{B}_{\mathrm{F}}$ in place of a $\beta(\Phi, \mathrm{B}(\Phi))$ where $\mathrm{B}(\Phi)$ is the variation of spacecraft elevation with longitude due to its motion during the scans. A linear interpolation is proposed, such as:

$$
\beta\left(\Phi, \mathrm{B}_{\mathrm{F}}\right)=\beta(\Phi, \mathrm{B}(\Phi))+\mathrm{a}_{\Phi} *\left(\mathrm{~B}_{\mathrm{F}}-\mathrm{B}(\Phi)\right)
$$

where the slope $a_{\Phi}$ is evaluated empirically from available data. In this way, the data reduction can be still pursued without any hypothesis on the ring layer structure. Figure 2 displays $\beta(B(\Phi))$ for all longitudes and observations. Its tortuous shape is bounded by the minimum $\beta$ values in its lower part (cyan and green points) and by the maximum $\beta$ values in its upper part (yellow and red points). Linear regression has been used to estimate coefficients $a_{\Phi}$ and $c_{\Phi}$ of $\beta(B(\Phi))=a_{\Phi} B+c_{\Phi}$ for various longitudinal ranges, corresponding either to minima, maxima or longitudes of intermediate wake amplitude. Two elevation ranges are considered for this fit, $|\mathrm{B}(\Phi)|<28^{\circ}$ or $|\mathrm{B}(\Phi)|>28^{\circ}$, corresponding to distinct datasets where $\mathrm{B}_{\mathrm{m}}<26^{\circ}$ and $\mathrm{B}_{\mathrm{m}}>34^{\circ}$. Error bars $\sigma_{\mathrm{a}}$ on $\mathrm{a}_{\Phi}$ slope are propagated to error bars on $\beta\left(\Phi, \mathrm{B}_{\mathrm{F}}\right)$.

Four azimuthal profiles of the quadrupolar asymmetry have been derived at reference elevations $\mathrm{B}_{\mathrm{F}}$ of $16^{\circ}, 20^{\circ}, 25^{\circ}$ and $36^{\circ}$ (Figure 3). The individual scans used to build a profile at elevation $\mathrm{B}_{\mathrm{F}}$ are indexed in Table 1. Before being summed, individual scans have been re-sampled azimuthally to one sample per degree. Within the new bin, measurements from different profiles are averaged. 
The bin error bar is the maximum of the dispersion around the average and the average error of individual measurements. The latter usually dominates. Two almost complete azimuthal profiles can be obtained at elevations $\mathrm{B}_{\mathrm{F}}=20^{\circ}$ and $\mathrm{B}_{\mathrm{F}}=36^{\circ}$ with all minima and maxima of the quadrupolar asymmetry visible (Fig. 3). Only partial information on the asymmetry is provided at $\mathrm{B}_{\mathrm{F}}=16^{\circ}$, which only gives the average $\beta$ factor for this elevation. Even if incomplete, the relative amplitude of the asymmetry is almost measurable at elevation $\mathrm{B}_{\mathrm{F}}=25^{\circ}$. It can be seen that the $\beta$ factors at maxima $\left(\Phi \sim 20^{\circ}\right.$ and $\left.\Phi \sim 200^{\circ}\right)$ are not significantly different within errors bars after this first correction. This is also true for minima $\left(\Phi \sim 110^{\circ}\right.$ and $\left.\Phi \sim 290^{\circ}\right)$. No difference appears then when self-gravity wakes are faced or seen from behind, along (minima) or perpendicularly (maxima) to their direction.

$* * * * * * * * * * * * * * *$ Insert Figure 3

\section{A ring self-gravity wakes' structure and vertical structure.}

Recent HST observations of the A ring quadrupolar asymmetry in visible light have been compared with ray-tracing calculation of the reflected light by simulated self-gravity wakes (French et al, 2007). Star occultation observations by UVIS and VIMS instruments in the ultraviolet and near-infrared domains have been fit to analytical models of self-gravity wakes, either considered as infinite elliptical cylinders or rectangular bars of infinite optical depth, interlaced with gaps filled with particles (Hedman et al. 2007, Colwell et al. 2006). This latter method has the advantage of simplifying the derivation of the shape and spacing of these 
temporal aggregates. The CIRS azimuthal scans are actually able to discriminate between both analytical models thanks to their almost complete sampling in azimuth.

3.1 Wakes models in the thermal infrared

The elliptical infinite cylinders wake model of Hedman et al. (2007) assumes that the self-gravity wakes are infinitely long, straight, parallel, regularly spaced with wavelength $\lambda$. They are opaque elliptical cylinders with finite height $\mathrm{H}$ and width $\mathrm{W}$. The optical depth in the gaps is $\tau_{\mathrm{G}}$. The pitch angle of the wakes relative to the local radial direction is noted $\Phi_{\mathrm{W}}$. The transmission function measured in star occultation at elevation B and longitude $\Phi$ is:

$$
T_{H}(\phi, B)=1-\sqrt{\left(\frac{H}{\lambda}\right)^{2} \frac{\sin ^{2}\left(\phi-\phi_{W}\right)}{\tan ^{2} B}+\left(\frac{W}{\lambda}\right)^{2}} e^{-\tau_{G} / \mu}=S_{G}(\phi, B) e^{-\tau_{G} / \mu}
$$

This can be read as the probability of a photon to be directly transmitted through a ring layer of optical depth $\tau_{\mathrm{G}}$ times its probability to effectively hit the gap, which projected fractional area as seen from $(\phi, B)$ is $\mathrm{S}_{\mathrm{G}}(\phi, \mathrm{B})$. This area is maximum as seen from above $\left(\mathrm{B}=90^{\circ}\right)$ or along the wakes direction $\Phi=\Phi_{\mathrm{W}}$ or $\Phi_{\mathrm{W}}+180^{\circ}$, and is noted $S_{\mathrm{H}, \mathrm{MAX}}$. It is minimum for $\Phi=\Phi_{\mathrm{W}} \pm 90^{\circ}$, and is noted $\mathrm{S}_{\mathrm{H}, \mathrm{MIN}}$, such as:

$$
S_{H, M I N}=1-\sqrt{\left(\frac{H}{\lambda \tan B}\right)^{2}+\left(\frac{W}{\lambda}\right)^{2}} \geq 0, S_{H, M A X}=1-\frac{W}{\lambda}
$$

The infinitely long and rectangular-shaped optically thick wakes offer a somewhat different transmission function (Colwell et al. 2007), which can be expressed as:

$$
T_{C}(\phi, B)=1-\frac{H}{\lambda} \frac{\left|\sin \left(\phi-\phi_{W}\right)\right|}{\tan B}+\frac{W}{\lambda} e^{-\tau_{G} / \mu}
$$


The maximum transmission of any stellar flux through the ring is $T_{M A X}(B)=S_{M A X} e^{-\tau_{G} / \mu}$ whatever the model is, but the minimum transmissions differ.

In a first attempt to model the thermal emission $I_{v}(\phi, B)$ of such a ring, we suppose here that it is isothermal, i.e. that there is no vertical temperature gradient and that both the wakes (of infinite optical depth) and the gaps have the same temperature. This is compatible with some CIRS observations of the A ring which shows identical temperatures on lit and unlit faces (Spilker et al. 2004). In some radial scans however, a 5K-large thermal gradient appears between lit and unlit faces (Spilker et al. 2006). The infrared spectrum of such a ring can be written:

$$
I_{v}(\phi, B)=\varepsilon B_{v}\left(T_{P}\right)\left(\left(1-e^{-\tau_{G} / \mu}\right) S_{G}+\left(1-S_{G}\right)\right)=\beta B_{v}\left(T_{P}\right)
$$

This sets up the relation between the CIRS $\beta$ factor and the transmission function:

$$
\beta=\varepsilon\left(1-S_{G}(\phi, B) e^{-\tau_{G} / \mu}\right)=\varepsilon(1-T)
$$

The minimum factor $\beta_{\min }=\varepsilon\left(1-\mathrm{S}_{\mathrm{MAX}} \mathrm{e}^{-\tau}{ }_{\mathrm{G}}{ }^{\mu}\right)$ is fixed by the gap ratio $\mathrm{W} / \lambda$ and the optical depth $\tau_{\mathrm{G}}$. Increasing the relative gap width requires decreasing its optical depth to fit a given $\beta_{\min }$. The observed relative amplitude of the asymmetry constrains the $\mathrm{H} / \lambda$ ratio, the stronger the contrast, the larger the ratio. The emissivity $\varepsilon$ is assumed to be unity in the wavelength range of the fit, as water ice, which is known to cover Saturn's ring particles, is highly absorbent in the mid- and farinfrared.

3.2 Shape and spacing of self-gravity wakes as seen by CIRS.

Observed $\beta$ factors are compared to the modelled factors as described above. Four different fits have been run and results are summarized in Table 2. The first two fits concern interpolated 
azimuthal profiles from varying viewing geometries and the last two compare raw data, as shown in figure 1 , to Hedman et al. or Colwell et al. models. The four structural fit parameters are $\mathrm{H} / \lambda$, $\mathrm{W} / \lambda, \Phi_{\mathrm{W}}$ and $\tau_{\mathrm{G}}$. Fits minimize chi-square with a Levenberg-Marquardt algorithm. First, the four interpolated profiles have been fitted individually to the Hedman's wake model. For one profile, fits are first run for the best correction factor $a_{\Phi}$ and then for $a_{\Phi} \pm \sigma_{\Phi}$, in order to include uncertainties induced by the interpolation method. The uncertainty on parameters is dominated by this uncertainty. Final error bars correspond to the standard deviation among the three values obtained with the three factors. Individually, profiles at elevations $\mathrm{B}_{\mathrm{F}}=20^{\circ}, 25^{\circ}$ and $36^{\circ}$ can only constrain the average pitch angle $\Phi_{\mathrm{W}}$ and the $\mathrm{H} / \lambda$ ratio as they contain minima and maxima. The "Av., int." in Table 2 shows the average values of the parameters obtained with these individual fits. Constrains on $\mathrm{W} / \lambda$ and $\tau_{\mathrm{G}}$ are weak. Second, the model is fitted to the merged data of the four profiles, with the same method to propagate the errors on $\mathrm{a}_{\Phi}$. Linking all geometries together highly constrains $W / \lambda$ and $\tau_{\mathrm{G}}$ which fix the average $\beta$ s relatively to one another. As the $H / \lambda$ ratio also influences the average $\beta$ factor, this ratio is also severely constrained in the global fit. Naturally the accuracy on the average pitch angle is improved as more minima and maxima are taken into account in the global fit. For each reference elevation $\mathrm{B}_{\mathrm{F}}$, this global best fit is plotted over CIRS data in figure 3. The reduced $\chi_{\mathrm{R}}^{2}$ is about 3.2. Next, the elliptical cylinder wake model is fit to the "raw" data as shown in figure 1, without the interpolation step. This provide a very close solution indeed, with a much better residual, $\chi_{\mathrm{R}}{ }^{2}=2.2$ as the largest dispersion due to uncertainty on $a_{\Phi}$ is no more present. Finally, we fitted the "raw" data with the rectangular bar wakes model. The residuals are significantly worse (Table 2) and structural parameters significantly differ from one model to the other, by $10 \%$ or more, except for the pitch angle. The $\mathrm{H} / \lambda$ ratio is most affected in the comparison as it controls the amplitude of the asymmetry, which 
shape highly differs between models (Figure 3). CIRS observations definitely favor the elliptical cylinder shape model for the A ring self-gravity wakes with structural parameters $H / \lambda=0.1607 \pm$ $0.0002, \Phi_{\mathrm{W}}=70.70 \pm 0.07^{\circ}, \mathrm{W} / \lambda=0.3833 \pm 0.0008$ and $\tau_{\mathrm{G}}=0.1231 \pm 0.0005$

$* * * * * * * * * * * * * * *$ Insert Table 2

3.3 Multi-wavelength view of self-gravity wakes in the A ring.

CIRS results can be first compared with VIMS and UVIS measurements by stellar occultation. The predicted maximum transmission $\mathrm{T}_{\mathrm{MAX}}$ derived from CIRS observations at VIMS geometry $\left(B=3.45^{\circ}\right)$ is $T_{\operatorname{MAX}}\left(B=3.45^{\circ}\right)=0.080 \pm 0.003$, i.e. the exact value measured by that instrument in the middle of the A ring (Hedman et al. 2007). It remains close to the UVIS estimation of $\mathrm{T}_{\mathrm{MAX}} \approx 0.09$ in this region (Colwell et al. 2006). This means that gap width-over-wavelength ratio $\mathrm{G} / \lambda=1-\mathrm{W} / \lambda=0.617$ measurements by VIMS and CIRS are identical if $\tau_{\mathrm{G}}(\mathrm{VIMS})=\tau_{\mathrm{G}}(\mathrm{CIRS})$. CIRS estimate of the average gap optical depth, i.e. $\tau_{\mathrm{G}}=0.1231$, is identical to UVIS measurement in the inner two-thirds of the A ring (Colwell et al. 2006). Without doubt averaging UVIS optical depth measurements over distance would lead to an accuracy on the gap optical depth comparable to CIRS one. All these together show that gap width and optical depth, derived by multiple instruments using very different methods at different wavelengths, are identical.

The height-over-wavelength ratio $\mathrm{H} / \lambda=0.160$ is of the same order, but significantly different from the VIMS estimate of 0.10 . But this latter value is somewhat dependent of a model which assumes an aspect ratio of $\mathrm{W} / \mathrm{H} \sim 1$, following results of numerical simulations (Salo et al. 2004). 
These same simulations provide $\mathrm{H} / \lambda$ ratios of $0.17-0.23$, very close to what is observed with CIRS. The vertical aspect ratio deduced from CIRS data is $\mathrm{H} / \mathrm{W} \approx 0.42$, (or $\mathrm{W} / \mathrm{H}=2.38$ ) is significantly higher than the UVIS ratio $(\mathrm{H} / \mathrm{W})_{\mathrm{UVIS}} \sim 0.2$ in the middle A ring, but with a slightly different wake model (Colwell et al. 2006). The fit of CIRS data with this model provides a value of $(\mathrm{H} / \mathrm{W}) \sim 0.22$, close to UVIS ratio. We may then expect CIRS and UVIS estimates of wakes structural parameters to be closer if they were adjusted to the same model. Elliptical cylinder wakes as seen by CIRS appear thicker compared to VIMS and UVIS views, then closer to the picture suggested by numerical simulations for volume density $\rho=450 \mathrm{~kg} \cdot \mathrm{m}^{-3}$ and surface mass density $\Sigma=500 \mathrm{~kg} \cdot \mathrm{m}^{-2}$ (Salo et al. 2004). CIRS results stay compatible with millimeter observations of direct Saturn light transmitted through the rings, i.e. $(\mathrm{H} / \lambda)_{\mathrm{mm}} \sim 0.2$ and $(\mathrm{H} / \mathrm{W})_{\mathrm{mm}} \sim$ 0.4 (Dunn et al. 2004). It is difficult to conclude at this stage whether self-gravitation is greater than expected to explain this effect, before a systematic study of morphological parameters against surface and particle volume densities has been conducted with the help of numerical simulations.

The CIRS measurement of the average pitch angle in the A ring, $\Phi_{\mathrm{W}}=70.70 \pm 0.07^{\circ}$, is in total agreement with that from VIMS. Also VIMS data averaged over distance in the inner A ring would provide similar accuracy on this average value. The discrepancy of UVIS pitch angle estimates, $\left[57^{\circ}-67^{\circ}\right]$ throughout the ring, with VIMS is confirmed by CIRS, but again they were determined with a slightly different wake model. Fit of CIRS data to Colwell et al model confirms the tendency toward a lower pitch angle when this model is used. CIRS value is compatible with the value of $70-75^{\circ}$ obtained from numerical simulations at a distance of $130,000 \mathrm{~km}$ form Saturn's center and particles volume density of $\rho=450 \mathrm{~kg} \cdot \mathrm{m}^{-3}$ (Salo et al. 2004). 
In normal viewing conditions $\left(\mathrm{B}=90^{\circ}\right)$, the azimuthal asymmetry should disappear and the expected $\beta$ value is 0.453 . The Cassini spacecraft will scan the A ring again at elevations of $50^{\circ}$ to- $70^{\circ}$ to more accurately estimate the normal ring optical depth. Expected azimuthal profiles are plotted on figure 3 .

$* * * * * * * * * * * * * * *$ Insert Figure 4

3.4 The vertical structure of the A ring.

The relative amplitude of the quadrupolar azimuthal asymmetry is usually defined as the quantity $A_{V}=2\left(I_{M A X}-I_{M I N}\right) /\left(I_{M A X}+I_{M I N}\right)$ where $I_{M A X}$ and $I_{M I N}$ are the observed maximum and minimum ring intensities at visual wavelengths (Thompson 1982, Franklin et al 1987). In this paper we define this same amplitude at infrared wavelengths as $A_{I}=2\left(\beta_{M A X}-\beta_{M I N}\right) /\left(\beta_{M A X}+\beta_{M I N}\right)$. Figure 4 displays $A_{I}$ versus observer elevation $B$ as measured from raw data (Figure 2) and from azimuthal profiles at the reference elevations $B_{F}$ (Figure 3), together with most significant $A_{V}$ measurements. CIRS "raw" points have been calculated with the $\beta_{\mathrm{MAX}}$ and $\beta_{\mathrm{MIN}}$ values colored in figure 2 . They have been interpolated from these values on a regularly-spaced elevation grid of one-degree-large bins. They provide systematically smaller relative amplitudes because interpolation is based on points that are not exactly at minimum (or maximum) $\beta$ for a given elevation (Figure 4). CIRS dots at $\mathrm{B}_{\mathrm{F}}$ elevations are calculated from the three azimuthal profiles of the $\beta$ factor at $20^{\circ}, 25^{\circ}$ and $36^{\circ}$. The fit with Hedman's model is in between these two clusters, exactly fitting the amplitude asymmetry at $\mathrm{B}_{\mathrm{F}}=20^{\circ}$. These discrepancies can be clearly identified in figure 3 too. 
CIRS best fit is clearly compatible with ground-based visual measurements at Earth elevation of $\sim 26^{\circ}$ (Thompson 1982, Salo et al. 2004). CIRS observations extend the measurement of the amplitude asymmetry to the $26^{\circ}-40^{\circ}$ elevation range where it continues on decreasing as expected. At $\mathrm{B}=38^{\circ}$, it is about $8 \pm 4 \%$. The constraints obtained with CIRS on the elliptical cylinder wake structure allow extrapolating the amplitude asymmetry at lower elevations. There is still a close agreement between infrared expectations and visual observations on the relative amplitude below $\mathrm{B}=16^{\circ}$. Ground-based and Voyager observations at elevations greater than $10^{\circ}$ are within the envelope of CIRS solution. The discrepancy is higher below $10^{\circ}$ for one HST measurement (French et al. 2007).

For a multilayer ring mixing gaps of optical $\tau_{\mathrm{G}}$ and wakes of width $\mathrm{W}$, height $\mathrm{H}$, infinite optical depth and spacing $\lambda$, the reflected light at visual wavelengths is expected to be, for ground-based observations:

$$
\frac{I_{S S, D=0}}{F}(\phi, B)=\frac{\mu_{0} \varpi_{0} P(\alpha)}{4\left(\mu+\mu_{0}\right)}\left(\left(1-e^{-2 \tau_{G} / \mu}\right) S_{G}(\phi, B)+\left(1-S_{G}(\phi, B)\right)\right)
$$

where $\mu_{0} \approx \mu$ is the sine of the solar elevation above the ring, $\omega_{0} \mathrm{P}(\alpha)$ are the mean scattering properties of the ring as a whole. The infrared-to-visible ratio of the relative amplitude of the asymmetry at elevation B should be in this context:

$$
\frac{A_{I}}{A_{V}}=\frac{1-\bar{S}_{G} e^{-2 \tau_{G} / \mu}}{1-\bar{S}_{G} e^{-\tau_{G} / \mu}} \times \frac{e^{-\tau_{G} / \mu}}{e^{-2 \tau_{G} / \mu}} \text { with } \bar{S}_{G}=\frac{1}{2}\left(S_{H, M I N}+S_{H, M A X}\right)
$$

This ratio is about 2 above $\mathrm{B}=10^{\circ}$ and rapidly increases below $10^{\circ}$. This is much larger than the observed ratio of about 1 (Figure 4). Salo and Karjalainen (2003) study of light scattering in rings of variable volume filling factor shows that a correction factor of $2 /\left(1+\exp \left(-\tau_{\mathrm{G}} / 2 \mu\right)\right)$ can be applied to equation (7), to mimic the increasing influence of shadow hiding between particles as 
the ring tends to a vertical monolayer structure and its volume filling factor D increases. In these conditions, the visible albedo of the ring is:

$$
\frac{I_{S S, \text { mono }}}{F}(\phi, B)=\frac{\varpi_{0} P(\alpha)}{4}\left(1-e^{-\tau_{G} / \mu} S_{G}(\phi, B)\right)
$$

and the ratio $A_{I} / A_{V}=1$, in perfect agreement with figure 4 . The comparison of CIRS with groundbased visible observations of the amplitude of the quadrupolar asymmetry, clearly shows that the A ring tends to a monolayer structure, with non-zero filling factor. Such a correction towards a monolayer structure already explained the reduced multiple scattering at large phase angles in this ring (Dones et al. 1993, Salo and Karjalainen 2003).

The critical wavelength of the Toomre instabilities, which self-gravity wakes actually are, is expected to be about $60 \mathrm{~m}$ at distance a=129,000 km for a surface mass density of $40 \mathrm{~g} \cdot \mathrm{cm}^{-2}$ (Salo et al. 2004, Tiscarino et al. 2007 for recent measurements). The height $\mathrm{H}$ and width $\mathrm{W}$ would be then about $10 \mathrm{~m}$ and $24 \mathrm{~m}$ respectively, values that fit the maximum particle sizes observed in this region by ground-based observations of stellar occultation (French and Nicholson 2000). The height is also below the thickness upper limit a ring would have if random velocities were isotropic, i.e. about $20 \mathrm{~m}$. As wakes attenuate random velocities perpendicular to the ring plane, a lower value for $\mathrm{H}$ is expected (Daisaka and Ida 1999, Tiscarino et al. 2007).

\section{Conclusions}

The CIRS campaign of azimuthal scans in the A ring has led to the first detection of the quadrupolar asymmetry in the thermal infrared domain. The so-called CIRS $\beta$ factor is found to 
be directly comparable with the ring filling factor. Multiple scans at different spacecraft elevations and over large azimuthal ranges put strong constraints on the average shape and spacing of self-gravity wakes in the inner two-third of the A ring. Its variations along azimuth strongly favors the elliptical cylinder wakes model (Hedman et al. 2007). Comparison of the relative amplitude of the quadrupolar asymmetry seen by the CIRS instrument with ground-based observations in the visible domain provides evidence that the A ring is a monolayer. These infrared observations yield an independent way of studying the wakes and confirm their ability to explore local dynamics of rings. The quality of the data set should benefit the calculation of filling factors from more "realistic" wakes, relying on time averaged spatial distributions of wakes provided by numerical simulations. After observations have constrained the wake structure, a systematic study of wake structure against key physical parameters like surface density, particle volume density and size distribution thanks to numerical simulations would be useful to constrain the physical conditions in the A ring. Beyond the study of the filling factor of wakes, the thermal modeling of their temperature should also provide strong insights on their structure, thermal properties and directional emissivity (Leyrat et al. 2007). The isothermal monolayer structure of this ring as proposed in this paper should also be tested against CIRS data.

\section{Acknowledgments}

This work was supported by the Programme National de Planétologie and the Centre National d'Etudes Spatiales. We thank both anonymous reviewers for helpful comments on this manuscript. It is dedicated to Jacques, to the precious time we shared, before he left. 


\section{References}

Altobelli, N., Spilker, L.J., Pilorz, S., Brooks, S., Edgington, S., Wallis, B., Flasar, M. 2007. C ring fine structure revealed in the thermal infrared. Icarus 191, 691-701.

Camichel, H. 1958. Mesures photométriques de Saturne et de son anneau. Ann. Astrophys. 21, 231-242.

Colombo, G., Goldreich, P., Harris, A.W. 1976. Spiral structure as an explanation for the asymmetric brightness of Saturn's A ring. Nature 264, 344-345.

Colwell, J.E., Esposito, L.W., Sremcevic, M. 2006. Self-gravity wakes in Saturn's A ring measured by stellar occultations from Cassini. Geophys. Res. Letters 33, L07201.

Colwell, J.E., Esposito, L.W., Sremcevic, M., Stewart, G.R., McClintock, W.E. 2007. Selfgravity wakes and radial structure in Saturn's B ring. Icarus 190, 127-144.

Daisaka, H., Ida, S. 1999. Spatial structure and coherent motion in dense planetary rings induced by self-gravitational instability. Earth Planets Space 51, 1195-1213.

Dones, L., Cuzzi, J.N., Showalter, M.R. 1993. Voyager photometry of Saturn's A ring. Icarus $105,184-215$.

Dunn, D.E., Molnar, L.A., Niehof, J.T., de Pater, I., Lissauer, J.J. 2004. Microwave observations of Saturn's rings: anisotropy indirectly and scattered saturnian thermal emission. Icarus $171,183-198$.

Flasar, M. and the CIRS Science Investigation Team 2004. Exploring the Saturn System in the thermal infrared: The Composite InfraRed Spectrometer. Space Sci. Rev. 115,169-297.

Ferrari, C., Spilker, L., Brooks, S., Edgington, S. G., Wallis, B., Pearl, J., Leyrat, C., Flasar, M., CIRS Investigation Team 2005. Azimuthal temperature variations in Saturn's rings as seen by the CIRS spectrometer onboard Cassini. Bull. Am. Astron. Soc. 37, 764. 
Ferrari, C., Leyrat, C. 2006. Thermal emission of spherical spinning ring particles. The standard model. Astron. Astroph. 447, 745-760.

Franklin, F.A., Cook, A.,F., Barrey, R.T.F, Roff, C.A., Hunt, G.E., de Rueda, H.B. 1987. Voyager observations of the azimuthal brightness variations of Saturn's rings. Icarus 69, 280-296.

French, R.G., Nicholson, P.D. 2000. Saturn's Rings II. Particle sizes inferred from stellar occultation data. Icarus 145 , p. 502-523.

French, R.G., Salo, H., McGhee C.A., Dones, L. 2007. HST observations of azimuthal asymmetry in Saturn's rings. Icarus 189, 493-522.

Froidevaux, L., Ingersoll, A.P. 1980. Temperatures and optical ddepths of Saturn's rings and a brightness temperature of Titan. J. Geophys. Res. 85, 5929-5936.

Hedman, M.M., Nicholson, P.D., Salo, H., Wallis, B.D., Buratti, B.J., Baines, K.H., Brown, R.H., Clark, R.N. 2007. Self-gravity wake structures in Saturn's A ring revealed by Cassini VIMS. Astron. J. 133, 2624-2629.

Lane, A.L. et al. 1982. Photopolarimetry from Voyager 2 - Preliminary results on Saturn, Titan and the rings. Science 215, 537-543.

Leyrat, C., Spilker, L. J., Altobelli, N., Pilorz, S., Ferrari, C., Edgington, S. G., Wallis, B. D., Nugent, C., Flasar, M. 2007. Infrared Observations Of Saturn's Rings: Azimuthal Variations And Thermal Modeling. AGU, abstract \#P43B-1295.

Leyrat, C., Spilker, L., Altobelli, N., Pilorz, S., Ferrari, C. 2008. Infrared observations of Saturn's rings by Cassini CIRS: Phase angle and local time dependence. Planet. Space. Sci. 56, 117133.

Lumme, K., Irvine, W.M. 1976. Photometry of Saturn's rings. Astron. J. 81, 863-893. 
Lumme, K., Esposito, L.W., Irvine, W.M., Baum, W.A. 1977. Azimuthal brightness variations of Saturn's rings: II- Observations at intermediate tilt angle. Astrophys. J. 216, L123-L126.

Lumme, K., Irvine, W.M. 1979. Azimuthal brightness variations of Saturn's rings: IIIObservations at tilt angle approximately equal to 11.5deg. Astrophys. J. 229, L109-L111.

Marouf, E., French, R.G., Rappaport, N.J., McGhee, C.A., Wong, K., Thompson, F.S. 2005. Eos Trans. AGU 85(47) AGU Fall Meet. Suppl. 86, P31D-04.

Nicholson, P.D., French, R.G., Campbell, D.B., Margot, J.-L., Nolan, M.C., Black, G.J., Salo, H. 2005. Radar Imaging of Saturn's rings. Icarus 177, 32-62.

Porco, C.C., Throop, H.B., Richardson, D.C. 2001. Light scattering in Saturn's rings: basic properties and the A ring azimuthal asymmetry. Bull. Am. Astron. Soc. 33, 1091.

Salo, H. 1992. Gravitational wakes in Saturn's rings. Nature 359, 619-621.

Salo, H. 1995. Simulations of dense planetary rings: III. Self-gravitating identical particles. Icarus $117,287-312$.

Salo, H., Karjalainen, R. 2003. Photometric modeling of Saturn's rings. I.Monte-Carlo method and the effect of non-zero filling factor. Icarus 164, 428-460.

Salo, H., Karjalainen, R., French, R.G. 2004. Photometric modeling of Saturn's rings. II. Azimuthal asymmetry in reflected and transmitted light. Icarus 170, 70-90.

Spilker, L., Ferrari, C., Cuzzi, J.N., Showalter, M., Pearl, J., Wallis, B. 2003. Saturn's rings in the thermal infrared. Planet. Space Sci. 51, 929-935.

Spilker, ～L., Pilorz, S. H., $\quad$ Edgington, S. G., $\quad$ Wallis, B. D., $\quad$ Brooks, S. M., $\quad$ Pearl, J. C., Flasar, F. M. 2005. Cassini CIRS Observations of a Roll-Off in Saturn Ring Spectra at Submillimeter Wavelengths. Earth, Moon, and Planets 96, 149-163.

Spilker, L.J., Pilorz, S.H., Wallis, B.D., Pearl, J.C., Cuzzi, J.N., Brooks, S.M., Altobelli, N., Edgington, S.G., Showalter, M.S., Flasar, F.M., Ferrari, C., Leyrat, C. 2006. Cassini 
thermal observations of Saturn's main rings: Implications for particle rotation and vertical mixing. Planet. Space Sci. 45, 1167-1176.

Thompson, W.T., Lumme, K., Irivine, W.M., Baum, W.A., Esposito, L.W. 1981. Saturn's rings azimuthal variations, phase curve, and radial profiles in four colors. Icarus 46, 187-200.

Thompson, W.T., 1982. The swarm model for the azimuthal brightness variations in Saturn's rings. Thesis, University of Massachusetts, Amherst.

Tiscarino, M.S., Burns, J.A., Nicholson, P.D., Hedman, M.M., Porco, C.C. 2007. Cassini imaging of Saturn's rings II. A wavelet technique for analysis of density waves and other radial structure in the rings. Icarus 189, 14-34.

Weiss, J.W., Porco, C. C., Richardson, D. C., Dones, L. 2006. Photometric Examination of Saturn's Rings as Seen in Cassini ISS Images. Lunar. Planet. Sci. Conf. XXXVII, 2371 (abstract). 


\begin{tabular}{|c|c|c|c|c|c|c|c|}
\hline Symbol & Start date & End date & $\begin{array}{l}\mathrm{B}^{\prime} \\
\left(^{\circ}\right)\end{array}$ & $\begin{array}{c}\mathrm{B}_{\mathrm{m}} \\
\left(^{\circ}\right)\end{array}$ & $\begin{array}{l}\alpha_{\mathrm{m}} \\
\left(^{\circ}\right)\end{array}$ & $\mathrm{r}_{\mathrm{SC}}$ & $\overline{\mathrm{B}_{\mathrm{F}}}$ \\
\hline+ & 2004-184T22:01:00 & 2004-185T00:42:00 & -24.5 & -16 & 104 & 24.9 & 16 \\
\hline *⿻丷木 & 2005-156T13:00:00 & 2005-156T21:00:00 & -21.5 & -19 & 39 & 25.7 & 20 \\
\hline 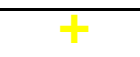 & 2005-178T00:44:00 & 2005-178T11:00:00 & -21.3 & 19 & 137 & 9.3 & 20 \\
\hline$\diamond$ & 2005-194T04:00:00 & 2005-194T08:00:00 & -21.1 & -21 & 24 & 19.0 & 20 \\
\hline+ & 2005-175T07:45:00 & 2005-175T13:00:00 & -21.3 & -20 & 39 & 22.7 & 20 \\
\hline *⿻丷木 & 2005-158T02:00:00 & 2005-158T13:00:00 & -21.5 & -22 & 20 & 14.6 & 20 \\
\hline+ & 2005-122T07:05:00 & 2005-122T13:35:00 & -21.8 & -26 & 15 & 9.9 & 25 \\
\hline$\diamond$ & 2007-114T06:45:00 & 2007-114T09:45:00 & -12.8 & 26 & 160 & 8.3 & 25 \\
\hline+ & 2007-128T13:15:00 & 2007-128T17:38:00 & -12.6 & 34 & 101 & 21.4 & 36 \\
\hline$*$ & 2007-048T16:53:00 & 2007-048T23:59:00 & -13.7 & -35 & 109 & 17.4 & 36 \\
\hline+ & 2007-129T17:00:00 & 2007-129T19:45:00 & -12.5 & 35 & 123 & 14.5 & 36 \\
\hline$\diamond$ & 2007-016T18:30:00 & 2007-016T22:04:54 & -14.7 & -37 & 24 & 12.7 & 36 \\
\hline+ & 2007-128T17:42:00 & 2007-128T19:45:00 & -12.6 & 37 & 96 & 22.2 & 36 \\
\hline
\end{tabular}

Table 1 - Azimuthal scans of the A ring executed with the CIRS spectrometer. Symbols refer to data plotted on figure 1. The solar elevation B', spacecraft elevation $B_{m}$ and phase angle $\alpha_{m}$ at ring intercept and the spacecraft distance to the planet $\mathrm{r}_{\mathrm{SC}}$ in units of Saturn radius, are the mean viewing geometries at the epoch of each scan. Elevations are negative for south lit face geometries. $\mathrm{B}_{\mathrm{F}}$ stands for the observer elevation at which the azimuthal scan has been empirically extrapolated to correct from varying viewing geometries during the measurements (section 2.3). 


\begin{tabular}{|c|c|c|c|c|c|c|}
\hline $\mathrm{B}_{\mathrm{F}}$ & $\mathrm{H} / \lambda$ & $\mathrm{W} / \lambda$ & $\Phi_{\mathrm{W}}\left(^{\circ}\right)$ & $\tau_{\mathrm{G}}$ & $\chi^{2}$ & $\chi_{\mathrm{R}}{ }^{2}$ \\
\hline Av., int., $\mathbf{H}$ & $0.13 \pm 0.03$ & $0.3 \pm 0.2$ & $71.1 \pm 1.5$ & $0.15 \pm 0.10$ & - & - \\
\hline All, int., $\mathbf{H}$ & $0.160 \pm 0.002$ & $0.383 \pm 0.003$ & $71.4 \pm 0.1$ & $0.123 \pm 0.003$ & 2602 & 3.2 \\
\hline All, "raw", H & $0.1607 \pm 0.0002$ & $0.3833 \pm 0.0008$ & $70.7 \pm 0.07$ & $0.1231 \pm 0.0005$ & 5085 & 2.2 \\
\hline All, "raw", C & $0.0746 \pm 0.0002$ & $0.3380 \pm 0.0008$ & $70.08 \pm 0.06$ & $0.1323 \pm 0.0005$ & 8929 & 3.9 \\
\hline
\end{tabular}

Table 2 - Fit results for wake structural parameters with CIRS data. The given pitch angle $\Phi_{\mathrm{W}}$ is the supplementary of the actual fit angle in equation (2), in order to be directly comparable with other studies. Fit are noted 'H' or 'C' when elliptical cylinder wake model (Hedman et al. 2007) or rectangular bars are used (Colwell et al. 2006, 2007), respectively. The 'Av., int.' row shows the average of fit results on the four interpolated profiles. 'All, int' corresponds to the best fit of the merged four azimuthal profiles interpolated at constant elevations $\mathrm{B}_{\mathrm{F}}$, with 820 data points. 'All, raw' corresponds to the best fit with raw data as shown in figure 1, with 2316 data points. 


\section{Figure captions}

Figure 1. Azimuthal variations of $\beta(\Phi, B)$ factor in the A ring. Mean spacecraft phase angle $\alpha_{m}$ and elevation $B_{m}$ relative to the ring intercept are given for each scan (see Table 1). Longitude $\Phi$ is the longitude of the spacecraft relative to the local radial direction at the ring intercept. For clarity, error bars have not been reported. There are about \pm 0.006 at $1 \sigma$ level of uncertainty. Vertical dotted lines mark longitudes of minima and maxima of the quadrupolar asymmetry as expected by numerical simulations.

Figure 2. Variations of $\beta(B(\Phi))$ factors with spacecraft elevation $|\mathrm{B}|$ in the A ring. Longitudes $\Phi$ are color-coded: cyan for minima with $\Phi \in\left[90^{\circ}-130^{\circ}\right] \mathrm{U}\left[280^{\circ}-310^{\circ}\right]$ for $|\mathrm{B}|<28^{\circ}$, green for minima with $\Phi \in\left[80^{\circ}-130^{\circ}\right] \mathrm{U}\left[270^{\circ}-310^{\circ}\right]$ for $|\mathrm{B}| \geq 28^{\circ}$, orange for maxima with $\Phi \in\left[0^{\circ}\right.$ $\left.50^{\circ}\right] \mathrm{U}\left[180^{\circ}-230^{\circ}\right]$ for $|\mathrm{B}|<28^{\circ}$, red for maxima with $\Phi \in\left[0^{\circ}-50^{\circ}\right] \mathrm{U}\left[170^{\circ}-210^{\circ}\right]$ for $|\mathrm{B}| \geq 28^{\circ}$, black for all the other longitudes. Straight lines correspond to best linear regression fits $\beta(\mathrm{B}(\Phi))=\mathrm{a}_{\Phi} \mathrm{B}+\mathrm{c}_{\Phi}$. Linear regression results $\left(\mathrm{a}_{\Phi}, \sigma_{\mathrm{a}}\right)$ are given here for completeness: (-7.09, $0.12) \times 10^{-3}$ for cyan line, $(-11.34,0.08) \times 10^{-3}$ for yellow line, $(-9.15,0.05) \times 10^{-3}$ for black line and $|\mathrm{B}|<28^{\circ},(-2.44,0.13) \times 10^{-3}$ for green line, $(-4.58,0.19) \times 10^{-3}$ for red line, $(-3.69,0.13) \times 10^{-3}$ for black line and $|\mathrm{B}| \geq 28^{\circ}$. For clarity error bars have not been reported. There are about \pm 0.006 at $1 \sigma$ level of uncertainty.

Figure 3. Averaged azimuthal profiles of the $A$ ring $\beta$ factor at $B_{F}=16^{\circ}, 20^{\circ}, 25^{\circ}$ and $36^{\circ}$ after correction for changing spacecraft elevation during the scans. Best fit for elliptical cylinders 
wake model $\mathrm{H}$ (dash-dot) or rectangular bars wake model $\mathrm{C}$ (dot) are superimposed with the same color. Corresponding structural parameters are given in the last two rows of Table 2. Expected profiles at elevations $\mathrm{B}=55^{\circ}$ for future CIRS observations of the A ring are also plotted. Errors bars are $\pm 1 \sigma$ standard deviation figuring dispersion between added scans and empirical correction from varying elevation.

\section{Figure 4.}

Amplitudes $A_{I}$ and $A_{V}$ of the quadrupolar asymmetry as a function of the observer elevation $B$ above the ring plane, as measured by CIRS in infrared or by ground-based (Thompson 1982), Voyager (Dones et al. 1993) or HST (French et al. 2007) in visible light. The HST measurement at $\mathrm{B}=20^{\circ}$ has been shifted by $0.5^{\circ}$ not to overlap CIRS one. CIRS data at reference elevations $\mathrm{B}_{\mathrm{F}}$ correspond to the measure of the relative amplitude asymmetry as seen in figure 3. CIRS raw data points correspond to measurements displayed in figure 2 interpolated at regularly spaced elevation samples. The full line represents the expected amplitude caused by elliptical cylinder wakes (Hedman et al 2007) with dimensions constrained by CIRS data. Uncertainties on this curve are within its thickness. 


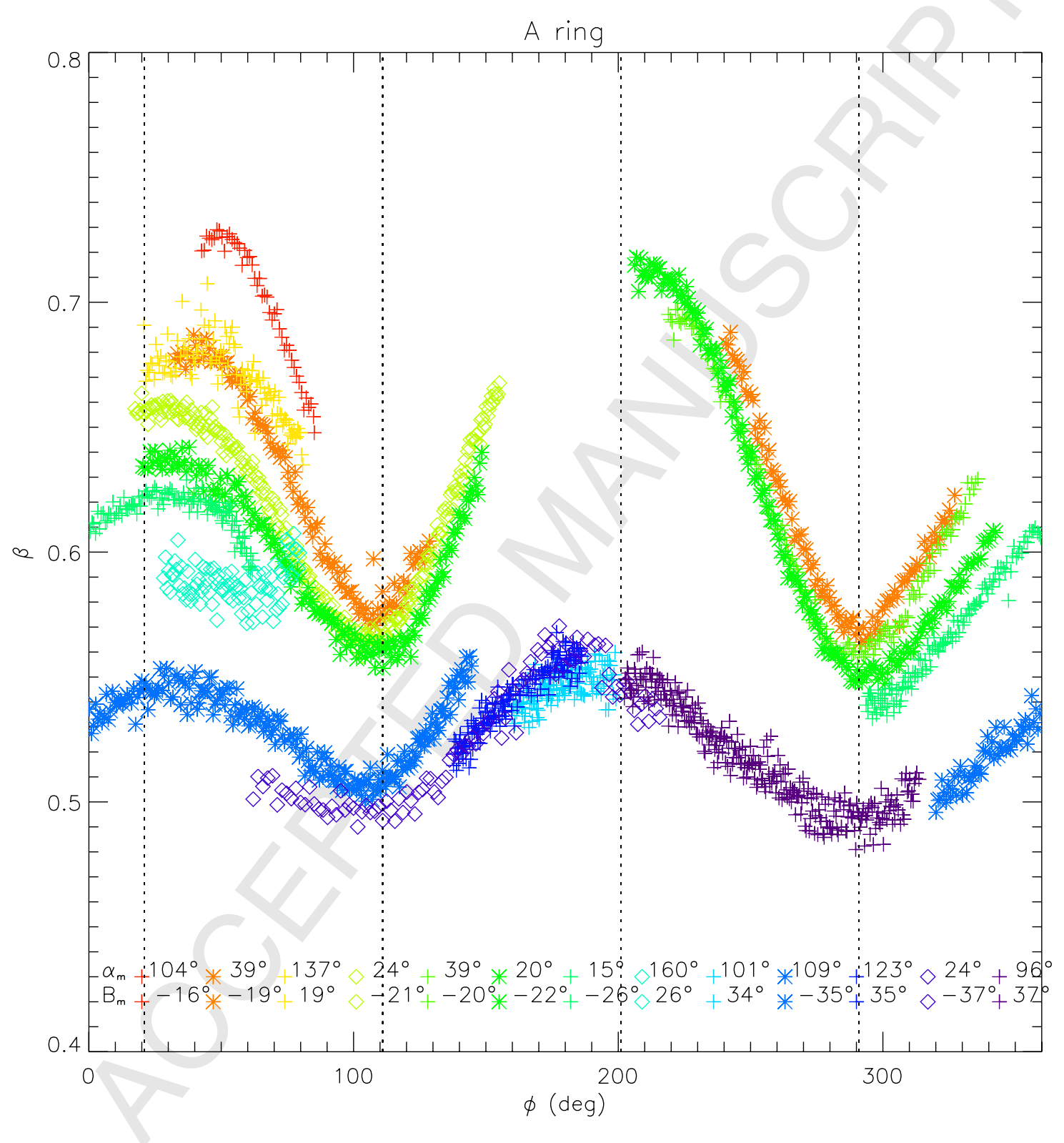




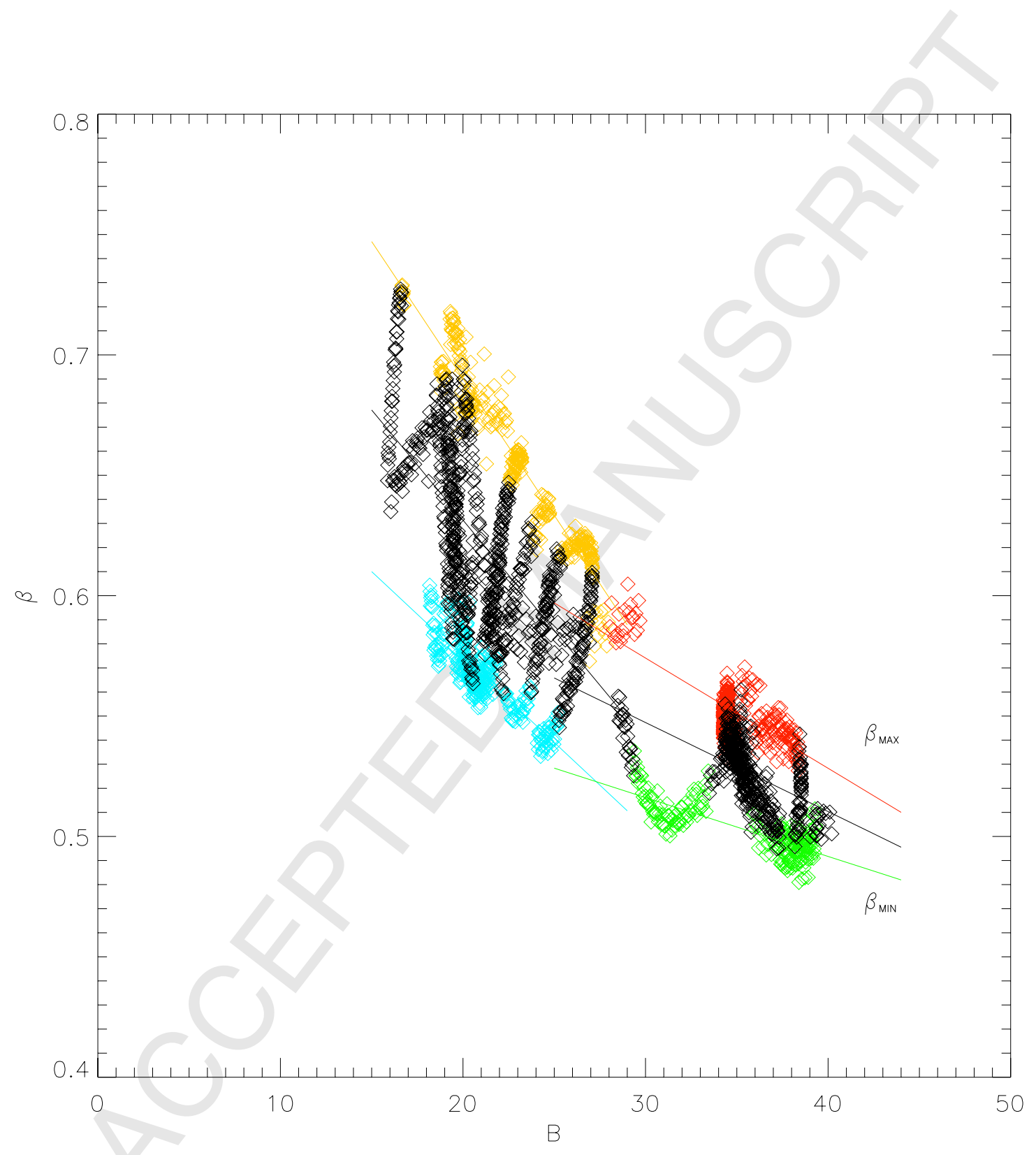




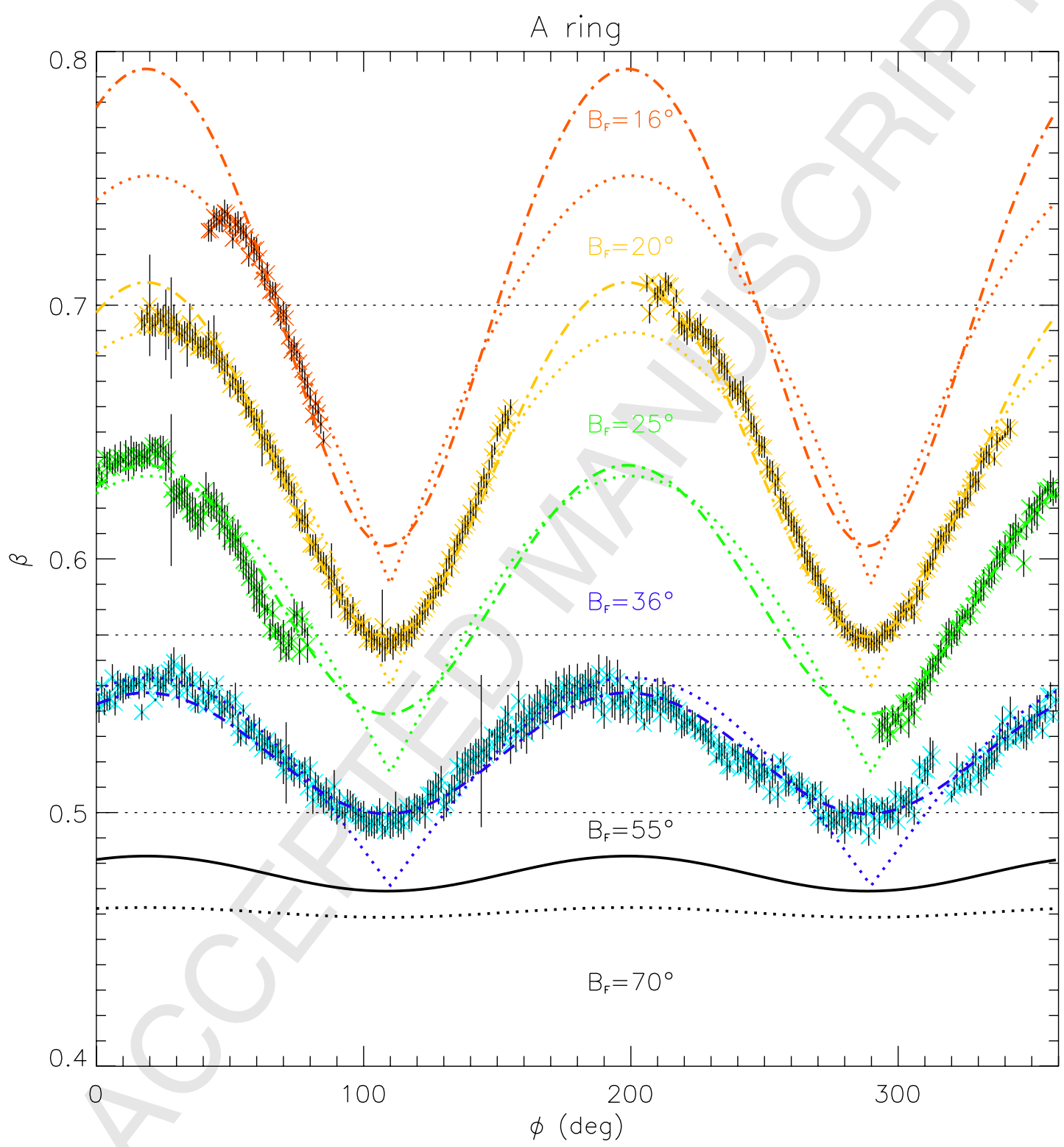




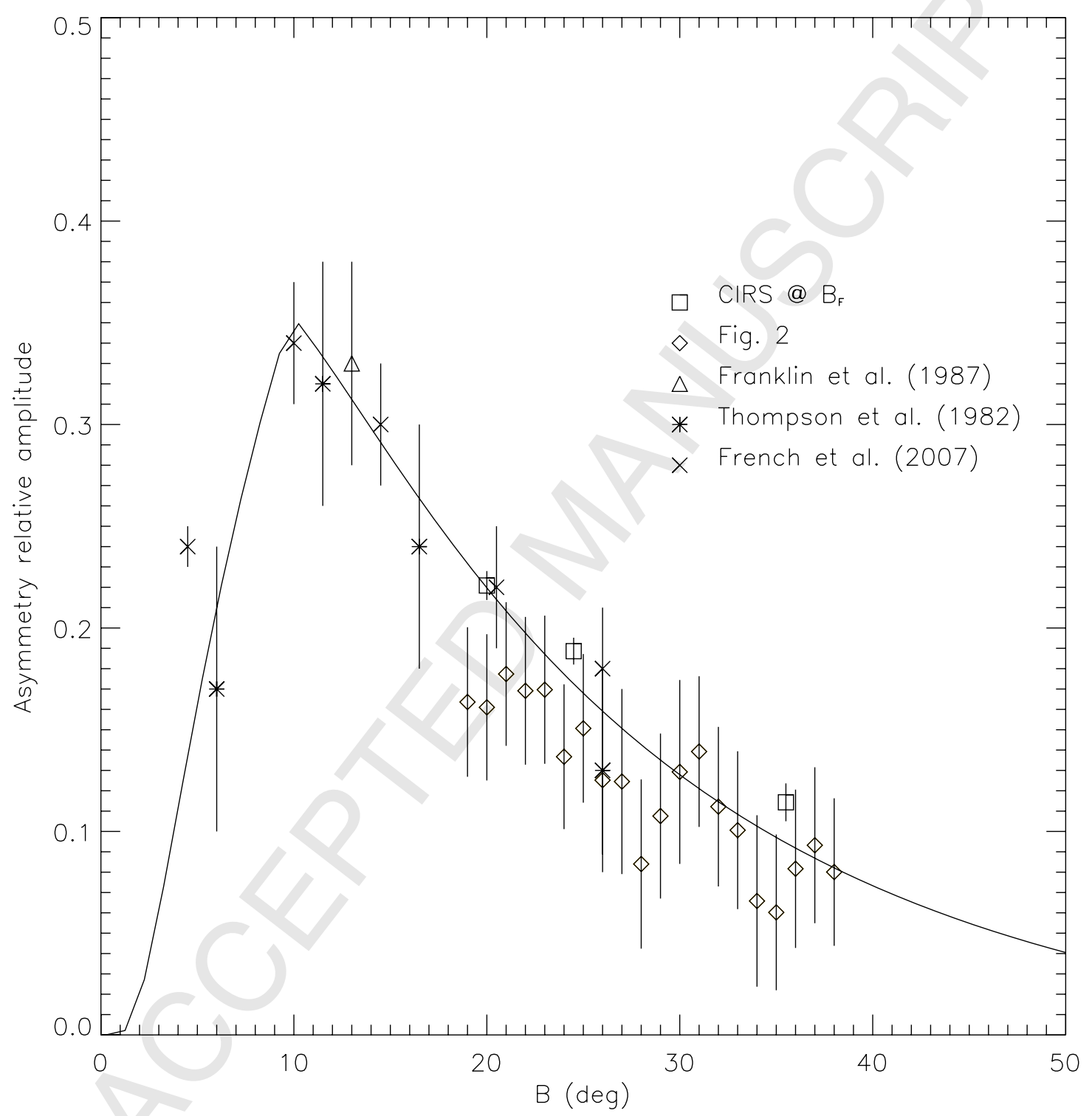

